\title{
ERITROGRAMA EM Oreochromis niloticus ALIMENTADAS COM NÍVEIS DE SORGO
}

\author{
MARTELINE, Marco Aurélio ${ }^{1}$ \\ DIAS, Luciana Thie Seki ${ }^{2}$ \\ PAIVA, Kelli Cristina ${ }^{3}$ \\ PESSA, Henderson ${ }^{4}$ \\ KIYUNA, Priscila Mieko Lima ${ }^{4}$ \\ SUSSEL, Fábio Rosa ${ }^{5}$
}

\begin{abstract}
RESUMO: O objetivo deste experimento foi avaliar a substituição do milho pelo sorgo e analisar os componentes hematológicos da série vermelha em tilápia do Nilo. Informações sobre parâmetros hematológicos são de grande valia, quando utilizados como indicadores do estado fisiológico e nutricional. Utilizou-se 40 tilápias machos com peso médio de $20 \mathrm{~g}$ criadas em tanques de $320 \mathrm{~L}$., alimentadas com rações isocalóricas e isoprotéicas de acordo com os níveis de substituição de 0,0;25,0;50,0; 75 e 100,0\%. Quinze peixes por tratamento foram anestesiados, pesados individualmente e o sangue retirado por punção cardíaca, com solução de EDTA (10\%), os parâmetros analisados foram: contagem do número de eritrócitos, taxa de hemoglobina e hematócrito. Com esses resultados foram calculados os índices hematimétricos. Foi utilizado o ANOVA e não houve diferença significativa entre os tratamentos, podendo concluir que o sorgo além de ser um potencial substituto do milho não altera os valores eritrocitários em Oreochromis niloticus.
\end{abstract}

Palavras-chaves: Sorgo. Hematologia. Tilápia.

\section{ERYTROGRAM Oreochromis niloticus FED WITH LEVELS OF SORGHUM}

\begin{abstract}
SUMMARY: The objective of this experiment was to evaluate the substitution of corn by sorghum and analyze the components of the red series hematology in Nile tilapia. Information on hematological parameters are of great value when used as indicators of physiological state and nutrition. We used 40 male tilapia with an average weight of $20 \mathrm{~g}$ reared in tanks of 320L., Fed diets isocaloric and isonitrogenous according to replacement levels of 0.0, 25.0, 50.0, 75 and 100.0\%. Fifteen fish per treatment were anesthetized, weighed individually and blood taken by cardiac puncture, with EDTA solution (10\%), the parameters analyzed were counting the number of erythrocytes, hemoglobin and hematocrit. With these results were calculated RBC indices. ANOVA was used and no significant difference between treatments can be concluded that sorghum in addition to being a potential substitute for corn does not change the values in erythroid Oreochromis niloticus.
\end{abstract}

Keywords: Sorghum, Hematology, Tilapia.

\footnotetext{
${ }^{1}$ Professor Titular do Centro Universitário Hermínio Ometto - UNIARARAS, Av Dr. Maximiliano Baruto, 500, Jd Universitário, Araras - SP - Brasil, CEP 13607-339, e-mail: marcomarteline@ hotmail.com

${ }^{2}$ Professora Adjunto do CCA/UFSCar, Rodovia Anhanguera, km174 CP 153 Araras - SP - Brasil, CEP13600970, e-mail: sekidias@ cca.ufscar.br

${ }^{3}$ Doutoranda do Programa de Alimentos e Nutrição da Faculdade de Ciências Farmacêuticas/UNESP, Rodovia Araraquara/Jaú, km, Araraquara - SP - Brasil, CEP 14801-902, e-mail: kcpaiva@ netsite.com.br

${ }^{4}$ Discente do Laboratório de Anatomia e Fisiologia Animal do Centro de Ciências Agrárias/UFSCar, Rodovia Anhanguera, km174 CP 153 Araras - SP - Brasil, CEP13600-970, e-mail: hendinho@hotmail.com e carlos_momo@uol.com.br

${ }_{5}^{5}$ Pesquisador Científico; Agencia Paulista de Tecnologia do Agronegócio Centro Leste - UPD Pirassununga, email: sussel@apta.sp.gov.br
} 


\section{INTRODUÇÃO}

A piscicultura é uma das atividades agroindustriais que mais se desenvolve no mundo. $\mathrm{O}$ Brasil é considerado um dos países com a maior perspectiva de crescimento, devido ao seu grande potencial hídrico (BORGHETTI et al. 2003).

A produção aqüícola brasileira entre a década de 90 e o ano 2000, teve um aumento de aproximadamente $900 \%$, passando de 20,5 mil para 210 mil toneladas (FAO, 2003). A produção brasileira somente na piscicultura continental em 2004, foi de aproximadamente 180 mil toneladas, as tilápias (Oreochromis niloticus), foram responsáveis por 40\% desse total, sendo uma das principais espécies cultivadas em todas as regiões do Brasil (IBAMA, 2005).

A tilápia do Nilo é considerada a segunda espécie de peixe mais criada no mundo, visto sua capacidade adaptativa aos atuais sistemas de produção (BORGHETTI et al. 2003), no entanto, se faz necessário a formulação de mais ações estratégicas para contornar as limitações técnicas e científicas.

A piscicultura intensiva tem necessidade de informações acuradas sobre a identificação e controle de enfermidades, que de modo geral, estão relacionadas às alterações do hemograma, por isso, o quadro hematológico vem sendo estudados, a fim de assegurar a saúde dos peixes. Assim, o conhecimento das variáveis hematológicas assume importância como meio auxiliar no diagnóstico. (TAVARES-DIAS et al. 2000b).

A orientação adequada, quanto à relevância das medidas nutricionais e profiláticas, para evitar a ocorrência de enfermidades, e conseqüentes perdas econômicas, faz-se necessária, visto que a nutrição correta e medidas preventivas são as melhores maneiras de evitar que o peixe adoeça ou deixe de crescer (TAVARES-DIAS; FAUSTINO, 1998).

O estudo das células sanguíneas assim como suas respectivas funções é importante para o conhecimento das condições de equilíbrio e patológicas. A detecção desses parâmetros pode auxilia na determinação da influência de condições fisiopatológicas que possam afetar a homeostase, contribuindo, no diagnóstico de condições adversas e na compreensão da relação entre os valores hematológicos e o estado de higidez dos peixes (TAVARES DIAS; MORAES, 1999, 2004).

Diante do exposto, este trabalho teve como objetivo avaliar os eritrogramas (hematócrito - Hct, número de eritrócito - Er e hemoglobina - $\mathrm{Hb}$ ) e os índices hematrimétricos (volume corpuscular médio - VCM; concentração de hemoglobina corpuscular média - CHCM; hemoglobina corpuscular média - HCM ) em Oreochromis niloticus alimentadas com níveis de sorgo de baixo tanino, substituindo o milho. 


\section{MATERIAL E MÉTODOS}

O experimento foi conduzido no Laboratório de Anatomia e Fisiologia Animal no Departamento de Biotecnologia Vegetal do Centro de Ciências Agrárias da Universidade Federal de São Carlos UFSCar- Campus de Araras (SP), com duração de 2 meses.

Foram utilizadas 400 tilápias do Nilo (Oreochromis niloticus) machos com peso médio inicial de $20 \mathrm{~g}$. Estes peixes foram cultivados em 5 tanques circulares com capacidade de 320L de água, independentes quanto à entrada e saída e saída de água e aeração.

O ciclo de iluminação adotado foi o de 12 horas de luz (natural e artificial) e 12 horas de escuro.

Os peixes foram alimentados com rações experimentais isocalóricas e isoprotéicas três vezes ao dia ad libitun, de acordo com os tratamentos experimentais:

a) Grupo controle - peixes alimentados com dieta comercial à base de milho e soja;

b) Grupo 1 - Substituição de $25 \%$ do milho da dieta por grãos de sorgo;

c) Grupo 2 - Substituição de 50\% do milho da dieta por grãos de sorgo;

d) Grupo 3 - Substituição de $75 \%$ do milho da dieta por grãos de sorgo;

e) Grupo 4 - Substituição de $100 \%$ do milho da dieta por grãos de sorgo.

Duas vezes ao dia as fezes e restos alimentares foram sifonadas e a água reposta imediatamente mantendo o nível de $310 \mathrm{~L}$ por tanque. Os valores médios de $\mathrm{pH}$ foram 6,5 \pm 0,3 e a concentração de oxigênio dissolvido $4,9 \pm 0,3 \mathrm{mg} / \mathrm{L}$.

Ao término do experimento os peixes ficaram 24 horas em jejum e após este período foram retirados ao acaso dez peixes de cada grupo experimental, anestesiados e envolvidos em pano umedecido, aproximadamente $2 \mathrm{~mL}$ de sangue foram coletadas por punção caudal e/ou cardíaca (JOSHI, 1977; MCKNICHT, 1996) em seringas com EDTA 0,1M.

Para confecção do hematócrito, a metodologia utilizada foi o do microhematócrito, segundo Glodenfarb et al. (1971), que consiste em preencher $3 / 4$ de dois microcapilares por amostra, e centrifugada a $12.500 \mathrm{rpm}$ por 5 minutos. A leitura foi realizada diretamente em escala padronizada, para a constatação do volume de células em relação ao volume total de sangue.

Para a contagem total de eritrócitos, as amostras foram homogeneizadas e diluídas 1:200 $(10 \mu \mathrm{L}$ de sangue total para $2 \mathrm{~mL}$ de solução fisiológica $0,9 \%)$. Depois de homogeneizar a solução, esperamos 5 minutos, cobrimos a câmara de Neubauer e utilizando a objetiva de 40x, foram contadas as células de cinco quadrados médios que foram multiplicados por 10000 sendo o número de eritrócitos, expresso em $10^{4} / \mathrm{mm}^{3}$. 
Para dosagem da hemoglobina o método utilizado foi o da cianometahemoglobina (COLLIER, 1944), que consiste em adicionar $10 \mu \mathrm{L}$ de sangue total em 2,5 mL de solução de Drabkin, a concentração de hemoglobina foi determinada pela leitura em espectrofotômetro, em comprimento de onda de 540nm.

Para precisão dos dados, as análises foram realizadas em duplicata para cada exemplar, sendo refeito quando a diferença entre elas foi igual ou superior a $20 \%$ (PITOMBEIRA, 1972; RANZANI-PAIVA 1995).

Com os resultados do hematócrito (Hct), do número de eritrócitos (Er) e da taxa de hemoglobina $(\mathrm{Hb})$ foram calculados os índices hematimétricos absolutos: VCM (Volume Corpuscular Médio) Ht x 10 / Er = fls; HCM (Hemoglobina Corpuscular Média) Hb x 10/ Er = picogramas; CHCM (Concentração de Hemoglobina Corpuscular Média) Hb x 100/ Ht = \% .

Para contagem diferencial de leucócitos, foram confeccionadas 4 lâminas para cada amostra após as extensões sanguíneas, foram coradas por May-Grünwald-Giensa, segundo Rosenfeld (1947).

Através do teste ANOVA, foram realizadas estatísticas de comparação entre os tratamentos para visualizar possíveis diferenças significativas que viessem ocorrer $(p>0,05)$.

\section{RESULTADOS}

As médias dos resultados da contagem de hemácias hemoglobina, hematócrito, volume corpuscular médio, concentração de hemoglobina corpuscular média, hemoglobina corpuscular média em $O$. niloticus, estão representados na Tabela 1.

Tabela 1. Valores médios de hemácias (HM), hemoglobina (HGB) e hematócrito (HCT), volume corpuscular médio (VCM), concentração de hemoglobina corpuscular média (CHCM), hemoglobina corpuscular média (HCM) de Oreochromis niloticus de acordo com os tratamentos utilizados.

\begin{tabular}{ccccccc}
\hline Tratamentos & $\begin{array}{c}\text { HM } \\
\left(\mathbf{1 0}^{\mathbf{6}} \boldsymbol{\mu L}\right)\end{array}$ & $\begin{array}{c}\text { HGB } \\
(\mathbf{g} / \mathbf{d L})\end{array}$ & $\begin{array}{c}\text { HCT } \\
(\boldsymbol{\%})\end{array}$ & $\begin{array}{c}\text { VCM } \\
(\mathbf{f L})\end{array}$ & $\begin{array}{c}\text { CHCM } \\
(\mathbf{g} / \mathbf{d L})\end{array}$ & $\begin{array}{c}\text { HCM } \\
(\boldsymbol{\mu g})\end{array}$ \\
\hline T1 & $1,77 \mathrm{AB}$ & $7,36 \mathrm{~B}$ & $23,88 \mathrm{~A}$ & $125,92 \mathrm{C}$ & $2,29 \mathrm{~A}$ & $33,69 \mathrm{C}$ \\
$\mathbf{T 2}$ & $1,57 \mathrm{~B}$ & $7,47 \mathrm{~B}$ & $26,96 \mathrm{~A}$ & $151,04 \mathrm{~B}$ & $2,66 \mathrm{~A}$ & $41,43 \mathrm{~B}$ \\
$\mathbf{T 3}$ & $1,65 \mathrm{AB}$ & $9,50 \mathrm{~A}$ & $27,22 \mathrm{~A}$ & $153,03 \mathrm{~B}$ & $2,69 \mathrm{~A}$ & $45,07 \mathrm{~B}$ \\
T4 & $2,35 \mathrm{~A}$ & $9,56 \mathrm{~A}$ & $27,01 \mathrm{~A}$ & $166,68 \mathrm{~A}$ & $2,62 \mathrm{~A}$ & $51,25 \mathrm{~A}$ \\
T5 & $1,52 \mathrm{~B}$ & $8,44 \mathrm{AB}$ & $25,62 \mathrm{~A}$ & $164,03 \mathrm{~A}$ & $2,56 \mathrm{~A}$ & $54,43 \mathrm{~A}$ \\
\hline DMS (5\%) & 0,72 & 1,78 & 3,55 & 8,86 & 0,79 & 5,32 \\
CV (\%) & 10,15 & 5,25 & 3,39 & 1,45 & 7,63 & 2,94 \\
\hline
\end{tabular}

T1 - tratamento com ração controle a base de milho e soja; T2 - tratamento com substituição de $25 \%$ do milho da ração por sorgo com baixo teor em taninos; T3 - tratamento com substituição de 50\% do milho da ração por sorgo com baixo teor em taninos; T4 - tratamento com substituição de $75 \%$ do milho da ração por sorgo com baixo teor em taninos; T5 - tratamento com substituição de $100 \%$ do milho da ração por sorgo com baixo teor em taninos.

Nucleus Animalium, v.2, n.2, nov.2010 


\section{DISCUSSÃO}

Utilizando-se do programa estatístico SAS, foi possível observar que ao nível de 5\% de probabilidade pelo Teste de Tukey, não houve variação significativa para hematócrito e concentração de hemoglobina corpuscular média.

Os valores obtidos de hematócrito correspondem aos descritos na literatura para a $O$. niloticus, sendo um indicador eficaz dos fatores ambientais a que os peixes estão expostos (TAVARES - DIAS; FAUSTINO, 1998).

Os valores médios de hemoglobina e CHCM, foram diferentes dos descritos para Oreochromis niloticus por Tavares-Dias e Faustino; (1998). Segundo Ranzani - Paiva (1991), a variação pode ocorrer devido a fatores exógenos como temperatura, estresse. $\mathrm{O}$ aumento do fluxo sanguíneo nas brânquias facilita as trocas gasosas, fornecendo mais oxigênio para o organismo, sendo uma resposta ao estresse (Mcdonald; Milligan, 1997).

De acordo com estudos realizados com tilápia vermelha (Oreochromis aureus) o sorgo pode ser incluído nas rações durante a fase de crescimento em 40\% (Castro et al.,1998).

A escolha pelo sorgo de baixo tanino é devido ao fato da formação de complexos que as proteínas e altos índices de taninos (acima de 0,65\%) podem formar, reduzindo a digestão da carboidratos, lipídios e proteínas (AUSTIN et al., 1989; MUELLER- HARVERY; McALLAN, 1992; FREIRE, 2002).

\section{CONCLUSÃO}

Nas condições em que o experimento foi realizado, pode-se concluir que:

A substituição do milho por sorgo de baixo tanino parcialmente ou totalmente, não influenciaram negativamente a hematolologia da Oreochromis niloticus.

\section{REFERÊNCIAS}

BORGHETTI, N.R.B.; OSTRENSKY, A.; BORGHETTI, J.R. Aquicultura: uma visão geral sobre a produção de organismos aquáticos no Brasil e no mundo. Curitiba: Grupo Integrado de Aqüicultura e Estudos Ambientais, 2003. 128p.

CASTRO, P. F. et al., Utilização de dietas a base de sorgo em rações de crescimento para tilápia vermelha (Oreochromis niloticus). In: AQUICULTURA BRASIL' 98,10,1998 Recife. Anais...Recife: ABRAq, 1998.p. 65- 72. 
FAO. El estado mundial de la pesca y la acuicultura. Disponivel em: www.fao.org. Acesso em: out. 2003.

FREIRE, E.S. Avaliação biológica de sorgo de alto e baio tanino por meio do desempenho e digestabilidade em tilápia do nilo (Oreochromis niloticus).2002. Dissertação (Mestrado de Zootecnia). Universidade Estadual Paulista, Botucatu.

IBAMA- Instituto Brasileiro Do Meio Ambiente E Dos Recursos Naturais Renováveis. Estatística da Pesca 2004. Brasília: IBAMA, 2005.136p.

RANZANI-PAIVA, M. J. T. Características hematológicas de tainha. Mugil platanus Gunther, 1880 (Osteichthyes, Mugilidae) da região estutário-laguna de Cananéia-SP. (Lat. 25000'S - Long. 47055'W). Boletim do Instituto de Pesca, v.22, n.1, p.1-22. 1995

RANZANI-PAIVA, M. J. T. Características sanguíneas da pirapitinga do sul, Brycon sp, sob condições experimentais de criação intensiva. Brazilian Journal of Veterinary Research and Animal Science, v.28, n.2, p.141-153. 1991.

ROSENFELD, G. Corante pancrômico para hematologia e citologia clínica: nova combinação dos componentes do May-Grunwald e do Giemsa num só corante de emprego rápido Mem Inst Butantan. Mem Inst Butantan. v. 20, p. 329-334, 1947.

TAVARES-DIAS, M. et al. Características hematológicas de teleósteos brasileiros. V. Variáveis do piauçu Leporinus macrocephalus Garavello e Britski, 1988 (Anostomidas). Naturalia, v.25, p.39-52, 2000 b.

TAVARES-DIAS, M. et al. Haematological changes in O. niloticus Linnaeus, 1758 with gill ichthyophthiriasis and saprolegniosis. Boletim do Instituto de Pesca, v.28, v.1, p.1-9; 2002.

TAVARES-DIAS, M.; FAUSTINO, C. D. Parâmetros hematológicos da tilápia-do-Nilo Oreochromis niloticus (Cichidae) em cultivo extensivo. Ars Veterinária, v.14, p.254-63, 1998.

TAVARES-DIAS, M.; MARTINS, M. L.; KRONKA, S. N. Evoluation of the haematological parameters in Piaractus mesopotamicus Holmberg ( Osteichthyes, Characidae) with Argulus $s p$. (Crustáceo, Branchiura) infestation and treatment with organophosphote. Rev. Brasileira Zoologia. v. 16, n. 2, p. 553-555, 1999.

TAVARES-DIAS, M.; MORAES, F. R. Hematologia de peixes Teleósteos. Ribeirão Preto: Lillimpress, 2004. 144p. 